\title{
Nitroalkanes in C-C Bond Forming Reactions: a Crystal Structure of a Complex of a Guanidine Catalyst and a Nitroalkane Substrate
}

\author{
Eddie van Aken, ${ }^{a}$ Hans Wynberg*, and Fré van Bolhuis ${ }^{b}$ \\ a Department of Organic Chemistry and ' Department of Crystallography, Nijenborgh 49747 AG Groningen, \\ The Netherlands
}

\begin{abstract}
van Aken, E., Wynberg, H. and van Bolhuis, F., 1993. Nitroalkanes in C-C Bond Forming Reactions: a Crystal Structure of a Complex of a Guanidine Catalyst and a Nitroalkane Substrate. - Acta Chem. Scand. 47: 122-124.

1,5,7-Triazabicyclo[4.4.0]dec-5-ene (TBD), a good catalyst for nitroalkanes in $\mathrm{C}-\mathrm{C}$ bond forming reactions, forms a $1: 1$ complex with phenylnitromethane as a substrate. The mode of complexation between the catalyst and the substrate is shown by a crystal structure 3 .
\end{abstract}

Dedicated to Professor Salo Gronowitz on the occasion of his 65 th birthday.

Nitroalkanes have been used surprisingly little in catalytic enantioselective $\mathrm{C}-\mathrm{C}$ bond forming reactions. ${ }^{1}$ This is in spite of the possibly short routes to optically active amines, ${ }^{2} \beta$-amino alcohols ${ }^{3}$ (e.g. ephedrine) and $\alpha$-amino acids. $^{4}$

Our first attempts using cinchona alkaloids ${ }^{1 \mathrm{a}}$ as chiral basic catalysts in Michael and Henry reactions, ${ }^{5}$ using nitroalkanes as the nucleophiles, resulted in slow conversions and low enantioselectivities in all reactions. The e.e.s did not exceed $25 \%$. The unknown mode of complexation between the cinchona alkaloids and the nitroalkanes hampered further development of these catalysts towards higher enantioselectivities.

In our view a predictable and well defined mode of complexation between the catalyst and the nitroalkane substrate is necessary towards directed improvements of the enantioselectivity of the catalyst. In the first step of a base-catalyzed $\mathrm{C}-\mathrm{C}$ bond forming reaction the achiral nitroalkane 1 is converted into the prochiral nitronate anion 2. In Scheme 1 this first step in the reaction is depicted.<smiles>[R]C([R])[N+](=O)[O-]</smiles>

1
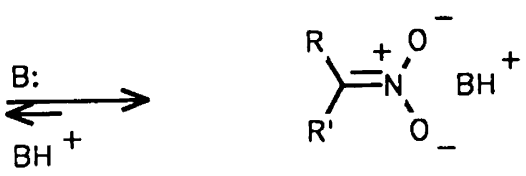

2
Scheme 1. The first step in a base-catalyzed addition reaction of nitroalkanes.

* To whom correspondence should be addressed.
The nitronate anion 2, a proposed intermediate in $\mathrm{C}-\mathrm{C}$ bond forming reactions, serves as an ideal handle for the catalyst. Fixation of this nitronate anion by the catalyst is essential because, at this stage of the reaction, the stereochemistry at $\mathrm{C}_{\alpha}$ of the product is determined. The catalyst should possess, after protonation, a structural element complementary to the structure of this nitronate anion 2 . The bicyclic guanidine, 1,5,7-triazabicyclo[4.4.0]dec-5-ene TBD, a strong base, possesses the right geometry after protonation. We used TBD as a model catalyst for testing the mode of complexation of bicyclic guanidines with nitroalkanes and its catalytic activity in $\mathrm{C}-\mathrm{C}$ bond forming reactions with nitroalkanes as the nucleophiles .

Enantioselective catalysis, for introducing chirality on $\mathrm{C}_{\alpha}$ of the nitroalkane, requires differentiation between the $R e$-site and the $S i$-site of the prochiral nitronate anion 2. This may eventually be achieved by using the reported chiral bicyclic guanidines. ${ }^{6}$

Here we report a crystal structure of a complex 3 of the achiral catalyst model, 1,5,7-triazabicyclo[4.4.0]dec5-ene TBD and phenylnitromethane (Fig. 1), establishing the mode of complexation between nitroalkane substrate and the guanidine catalyst.

The bicyclic guanidine TBD is an excellent catalyst for Michael and Henry reactions of nitroalkanes in toluene. ${ }^{7}$ An example is the Michael addition of nitroethane to methyl vinyl ketone explored in a $0.1 \mathrm{M}$ reaction using $10 \%$ TBD as the catalyst. This afforded $75 \% 5$-nitro2-hexanone within $5 \mathrm{~min}$. With quinine as the catalyst only $75 \%$ of 5-nitro-2-hexanone is formed after $122 \mathrm{~h}$ of reaction time; the enantioselectivity of the product was $13 \%$. 


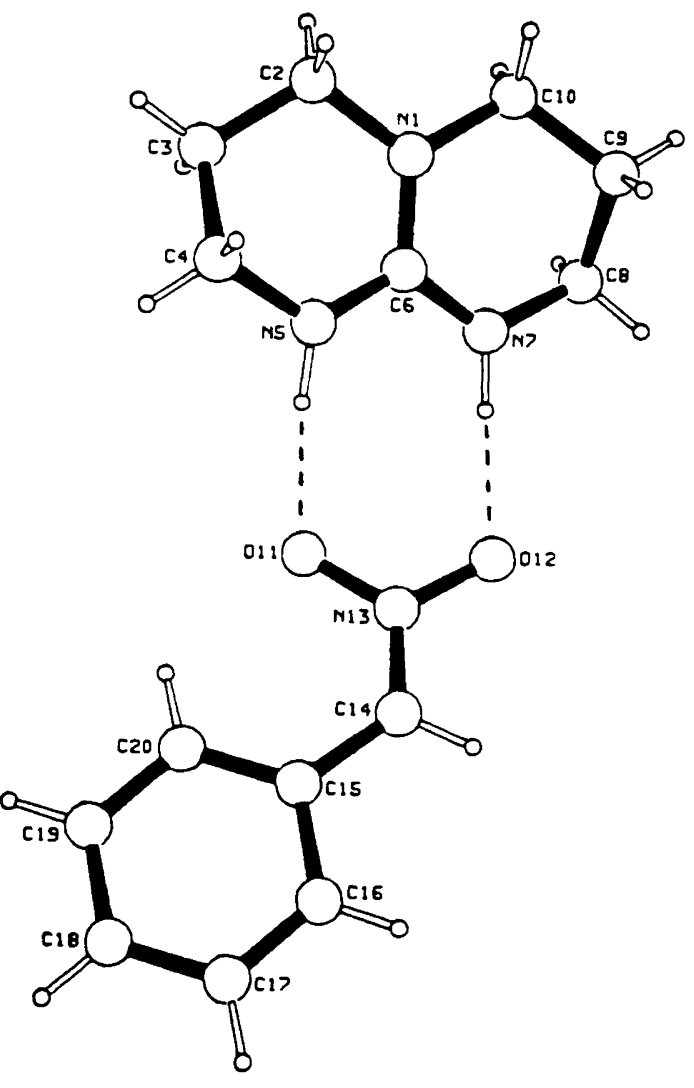

3

Fig. 1. The crystal structure of the salt 3 of 1,5,7-triazabicyclo[4.4.0] dec-5-ene (TBD) and phenylnitromethane showing the atomic numbering scheme. The two $\mathrm{H} \cdots \mathrm{O}$ distances: $H(5)-O(11) 1.97(3)$ and $H(7)-O(12) 1.99 \AA$ indicate real hydrogen bonds.
NITROALKANES IN C - C BOND FORMING REACTIONS

Table 1. The final positional parameters and their standard deviations of the bicyclic guanidinium/nitronate salt $\mathbf{3}$.

\begin{tabular}{lllll}
\hline Atom & $x$ & $y$ & $z$ & $B(A 2)$ \\
\hline 011 & $0.7941(2)$ & $0.5991(2)$ & $0.2379(1)$ & $2.13(4)$ \\
O12 & $0.6114(2)$ & $0.5825(2)$ & $0.1374(1)$ & $2.06(3)$ \\
N1 & $0.7734(2)$ & $0.1507(2)$ & $0.1535(1)$ & $1.70(4)$ \\
N5 & $0.8080(2)$ & $0.3368(2)$ & $0.2445(2)$ & $1.92(4)$ \\
N7 & $0.6922(2)$ & $0.3412(2)$ & $0.0904(2)$ & $1.99(4)$ \\
N13 & $0.6774(2)$ & $0.6410(2)$ & $0.2118(1)$ & $1.61(4)$ \\
C2 & $0.8616(3)$ & $0.0782(2)$ & $0.2235(2)$ & $2.24(5)$ \\
C3 & $0.9525(3)$ & $0.1629(3)$ & $0.2865(2)$ & $2.60(6)$ \\
C4 & $0.8750(3)$ & $0.2707(2)$ & $0.3296(2)$ & $2.33(5)$ \\
C6 & $0.7581(2)$ & $0.2758(2)$ & $0.1624(2)$ & $1.53(5)$ \\
C8 & $0.6397(3)$ & $0.2828(2)$ & $-0.0040(2)$ & $2.46(6)$ \\
C9 & $0.5939(3)$ & $0.1503(2)$ & $0.0194(2)$ & $2.60(6)$ \\
C10 & $0.7068(3)$ & $0.0784(2)$ & $0.0707(2)$ & $2.38(5)$ \\
C14 & $0.6247(2)$ & $0.7390(2)$ & $0.2557(2)$ & $1.62(5)$ \\
C15 & $0.6842(2)$ & $0.8151(2)$ & $0.3379(2)$ & $1.49(4)$ \\
C16 & $0.6104(2)$ & $0.9174(2)$ & $0.3724(2)$ & $1.76(5)$ \\
C17 & $0.6599(2)$ & $0.9960(2)$ & $0.4489(2)$ & $1.93(5)$ \\
C18 & $0.7840(2)$ & $0.9774(2)$ & $0.4939(2)$ & $2.08(5)$ \\
C19 & $0.8584(2)$ & $0.8779(2)$ & $0.4606(2)$ & $2.06(5)$ \\
C20 & $0.8099(2)$ & $0.7970(2)$ & $0.3845(2)$ & $1.71(5)$ \\
H5 & $0.807(3)$ & $0.415(3)$ & $0.241(2)$ & $* * * * *$ \\
H7 & $0.675(3)$ & $0.411(3)$ & $0.105(2)$ & $* * * * *$ \\
\hline
\end{tabular}

\section{Experimental}

General methods. The NMR spectra were recorded using a Varian VXR-300 at ambient temperature. Deuteriated benzene was used as the solvent. The addition reactions were performed in dry toluene; the products were isolated by filtration of the reaction mixture over silica gel (Merck silica gel/60) followed by evaporation of the solvent and excess reagents.

Table 2. The bond lengths $(\AA)$ and angles $\left({ }^{\circ}\right)$ of $\mathbf{3}$ and their estimated standard deviations.

\begin{tabular}{|c|c|c|c|c|c|c|c|c|c|c|c|}
\hline Atom 1 & \multicolumn{2}{|c|}{ Atom 2} & Distance & \multicolumn{2}{|l|}{ Atom 1} & Atom 2 & Distance & \multicolumn{2}{|l|}{ Atom 1} & Atom 2 & Distance \\
\hline $\begin{array}{l}\text { O11 } \\
\text { O12 } \\
\text { N1 } \\
\text { N1 } \\
\text { N1 } \\
\text { N5 } \\
\text { N5 } \\
\text { N5 } \\
\text { N7 }\end{array}$ & $\begin{array}{l}\text { N13 } \\
\text { N13 } \\
\text { C2 } \\
\text { C6 } \\
\text { C10 } \\
\text { C4 } \\
\text { C6 } \\
\text { H5 } \\
\text { C6 }\end{array}$ & & $\begin{array}{l}310(2) \\
314(2) \\
472(3) \\
344(3) \\
470(3) \\
464(3) \\
337(3) \\
83(3) \\
329(3)\end{array}$ & $\begin{array}{l}\text { N7 } \\
\text { N7 } \\
\text { N13 } \\
\text { C2 } \\
\text { C3 } \\
\text { C8 } \\
\text { C9 } \\
\text { C14 } \\
\text { C15 }\end{array}$ & $\begin{array}{l}\text { C8 } \\
\mathrm{H} 7 \\
\text { C1 } \\
\text { C3 } \\
\text { C4 } \\
\text { C9 } \\
\text { C1 } \\
\text { C1 } \\
\text { C1 }\end{array}$ & & $\begin{array}{l}1.465(3) \\
0.79(3) \\
1.327(3) \\
1.510(4) \\
1.527(4) \\
1.524(4) \\
1.516(4) \\
1.457(3) \\
1.421(3)\end{array}$ & $\begin{array}{l}\text { C15 } \\
\text { C16 } \\
\text { C17 } \\
\text { C18 } \\
\text { C19 } \\
011 \\
012 \\
011 \\
012\end{array}$ & & & $\begin{array}{l}1.413(3) \\
1.383(3) \\
1.391(3) \\
1.397(3) \\
1.390(3) \\
2.792(2) \\
2.781(2) \\
1.97(3) \\
1.99(3)\end{array}$ \\
\hline Atom 1 & Atom 2 & Atom 3 & Angle & Atom 1 & Atom 2 & Atom 3 & Angle & Atom 1 & Atom 2 & Atom 3 & Angle \\
\hline $\begin{array}{l}\mathrm{C} 2 \\
\mathrm{C} 2 \\
\mathrm{C} 6 \\
\mathrm{C} 4 \\
\mathrm{C} 4 \\
\mathrm{C} 6 \\
\mathrm{C} 6 \\
\mathrm{C} 6 \\
\mathrm{C} 8 \\
011\end{array}$ & $\begin{array}{l}\text { N1 } \\
\text { N1 } \\
\text { N1 } \\
\text { N5 } \\
\text { N5 } \\
\text { N5 } \\
\text { N7 } \\
\text { N7 } \\
\text { N7 } \\
\text { N13 }\end{array}$ & $\begin{array}{l}\text { C6 } \\
\text { C10 } \\
\text { C10 } \\
\text { C6 } \\
\mathrm{H} 5 \\
\mathrm{H} 5 \\
\mathrm{C} 8 \\
\mathrm{H} 7 \\
\mathrm{H} 7 \\
\mathrm{O} 12\end{array}$ & $\begin{array}{l}122.3(2) \\
115.6(2) \\
122.0(2) \\
121.8(2) \\
122 .(2) \\
116 .(2) \\
122.1(2) \\
115 .(2) \\
122 .(2) \\
117.2(2)\end{array}$ & $\begin{array}{l}\text { O11 } \\
\text { O12 } \\
\text { N1 } \\
\text { C2 } \\
\text { N5 } \\
\text { N1 } \\
\text { N1 } \\
\text { N5 } \\
\text { N7 } \\
\text { C8 }\end{array}$ & $\begin{array}{l}\text { N13 } \\
\text { N13 } \\
\text { C2 } \\
\text { C3 } \\
\text { C4 } \\
\text { C6 } \\
\text { C6 } \\
\text { C6 } \\
\text { C8 } \\
\text { C9 }\end{array}$ & $\begin{array}{l}\text { C14 } \\
\text { C14 } \\
\text { C3 } \\
\text { C4 } \\
\text { C3 } \\
\text { N5 } \\
\text { N7 } \\
\text { N7 } \\
\text { C9 } \\
\text { C10 }\end{array}$ & $\begin{array}{l}123.4(2) \\
119.5(2) \\
111.7(2) \\
109.2(2) \\
107.8(2) \\
120.6(2) \\
120.8(2) \\
118.6(2) \\
108.7(2) \\
108.4(2)\end{array}$ & $\begin{array}{l}\text { N1 } \\
\text { N13 } \\
\text { C14 } \\
\text { C14 } \\
\text { C16 } \\
\text { C15 } \\
\text { C16 } \\
\text { C17 } \\
\text { C18 } \\
\text { C15 }\end{array}$ & $\begin{array}{l}\text { C10 } \\
\text { C14 } \\
\text { C15 } \\
\text { C15 } \\
\text { C15 } \\
\text { C16 } \\
\text { C17 } \\
\text { C18 } \\
\text { C19 } \\
\text { C20 }\end{array}$ & $\begin{array}{l}\text { C9 } \\
\text { C15 } \\
\text { C16 } \\
\text { C20 } \\
\text { C20 } \\
\text { C17 } \\
\text { C18 } \\
\text { C19 } \\
\text { C20 } \\
\text { C19 }\end{array}$ & $\begin{array}{l}112.1(2) \\
126.6(2) \\
117.1(2) \\
125.4(2) \\
117.5(2) \\
121.1(2) \\
120.8(2) \\
119.0(2) \\
121.1(2) \\
120.5(2)\end{array}$ \\
\hline
\end{tabular}


Single crystals of $3,4,6,7,8,9$-hexahydro- $2 \mathrm{H}$-pyrimido[1,2-a]pyrimidine phenylnitronate 3 were obtained from a solvent mixture of toluene and pentane. The crystal structure of the complex 3 shows a dihydrogen bridge bonding mode between the substrate and the catalyst. The essential hydrogen bonding atoms $\mathrm{H} 5$ and $\mathrm{H} 7$, respectively, were refined individually. The $\mathrm{H}$... O distances of, respectively, $1.97 \AA$ and $1.99 \AA$ indicate real hydrogen bonds. $^{8}$

Crystal data for 3,4,6,7,8,9-hexahydro- $2 \mathrm{H}$-pyrimido[1,2-a]pyrimidine phenylnitronate $3, \mathrm{C}_{7} \mathrm{H}_{14} \mathrm{~N}_{3}$. $\mathrm{C}_{7} \mathrm{H}_{6} \mathrm{NO}_{2}, M_{\mathrm{w}}=276.34$, were collected at $130 \mathrm{~K}$, to give the following data: monoclinic, space group $P 2_{1} / a$, $a=10.338(2), b=10.627(2), c=13,232(3) \AA, \beta=93.95(2)^{\circ}$ and $V=1456.2 \AA .^{3}$ For $Z=4$ and $F_{\mathrm{w}}=276.34$ the $D_{\mathrm{c}}=1.266 \mathrm{~g} \mathrm{~cm}^{-3} \cdot \lambda=0.71073 \AA, \mu\left(\mathrm{Mo}-K_{\alpha}\right)=0.82 \mathrm{~cm}^{-1}$. The final $R$ value was $R=0.041$ and $w_{\mathrm{R}}=0.048$ for 2470 reflections. The final atomic parameters and their estimated standard deviations for $\mathbf{3}$ are given in Table 1, the bond lengths and angles in Table 2.

\section{Results and discussion}

The dihedral angle between the guanidine plane $\mathrm{N} 5-\mathrm{C} 6-\mathrm{N} 7$ and the nitronate plane $\mathrm{O} 11-\mathrm{N} 13-\mathrm{O} 12$ is $39.6^{\circ}$. The 'head to head' orientation in 3 satisfies the demand for a tight and predictable complexation between the catalyst and the nitroalkane.

NMR spectroscopy studies $\left({ }^{1} \mathrm{H},{ }^{13} \mathrm{C}\right.$ and $2 \mathrm{D}$ NOE experiments) also suggested a 'head to head' orientation of the two ionic components in solution. $300 \mathrm{MHz}{ }^{1} \mathrm{H}$ NMR spectroscopy of a $1: 1$ mixture of TBD and phenylnitromethane in benzene- $d_{6}$ showed complete deprotonation of phenylnitromethane. The signal of the $\mathrm{C}_{\alpha}$-methylene group $(\delta=5.43, \mathrm{~s})$ disappeared from the spectrum. The remaining $\mathrm{C}_{\alpha}-\mathrm{H}$ shifted into the aromatic region $(\delta=7.56, \mathrm{~s})$. A mixture of $1: 2$ of TBD and phenylnitromethane in benzene- $d_{6}$ showed two distinct pairs of signals for the deprotonated and complexed nitronate anion and the free phenylnitromethane. ${ }^{13} \mathrm{C}$ NMR showed distinct signals for all six aromatic carbon atoms in the $1: 1$ mixture, indicating a relatively slow rotation of the phenyl group, due to conjugation with the $\mathrm{C}=\mathrm{N}$ double bond. 2D NOE experiments in benzene- $d_{6}$, revealed no interaction between any of the hydrogens of the two ionic components, ruling out a stacked or sandwich structure of the complex in solution.

The crystal structure 3 is, to our knowledge, the first example of a complex of a basic catalyst and a nitroalkane substrate. It combines the two aspects of recognition and catalysis. The type of complexation is analogous to the complexation of carboxylate, ${ }^{9}$ phosphate, ${ }^{9 a, 10}$ and nitrate ${ }^{11}$ anions by the guanidinium moiety. The reported complexation of a nitrate anion by Schmidtchen ${ }^{11}$ showed a similar binding mode.
Experiments with the chiral byclic guanidine, placed at our disposal by Prof. J. de Mendoza, are currently under investigation and will be published in due course.

Acknowledgments. The work was supported by the Dutch Foundation for Chemical Research (SON), with financial aid from the Dutch Organization for Scientific Research (NWO).

\section{References}

1. (a) Wynberg, H. and Helder, R. Tetrahedron Lett. (1975) 4057; (b) Colonna, S., Hiemstra, H. and Wynberg, H. J. Chem. Soc., Chem. Commun. (1978) 238; (c) Matsumoto, K. and Uchida, T. Chem. Lett. (1981) 1673; (d) Sera, A., Takagi, K., Katayama, H., Yamada, H. and Matsumoto, K. J. Org. Chem. 53 (1988) 1157; (e) Schionato, A., Paganelli, S., Botteghi, C. and Chelucci, G. J. Mol. Catal. 50 (1989) 11.

2. Kornblum, N. and Fishbein, L. J. Am. Chem. Soc. 77 (1955) 6266.

3. Barrett, A. G. M. and Spilling, C. D. Tetrahedron Lett. 29 (1988) 5733.

4. Seebach, D., Häner, R. and Vettiger, T. Helv. Chim. Acta 70 (1987) 1507.

5. An example of a Henry reaction is given. Nitroethane is reacted with $o$-chlorobenzaldehyde in a $0.3 \mathrm{M}$ solution, using $10 \%$ quinidine as the chiral catalyst, resulting in a mixture of four diastereoisomers. The diastereomeric excess and both the enantiomeric excesses varied with time. The reaction was worked up after $60 \%$ conversion was obtained. The d.e. of the reaction was $40 \%$ (according to ${ }^{1} \mathrm{H}$ NMR spectroscopy). The two diastereoisomers were separated by chromatography (silica gel $\mathrm{CH}_{2} \mathrm{CCl}_{2} / 1 \% \mathrm{Et}_{3} \mathrm{~N}$ ). The e.e.s were $13 \%$ for the threo and $10 \%$ for the erythro diastereoisomer. The e.e.s were determined by derivatizing the $\beta$-nitro alcohols with Mosher's reagent [Dale, J. A., Dull, D. L. and Mosher, H. S. J. Org. Chem. 34 (1969) 2543] and by ${ }^{19} \mathrm{~F}$ NMR spectroscopy. This Henry reaction is, as far as we know, the first enantioselective catalytic example reported.

6. A chiral bicyclic guanidine, an analog of TBD, has already been synthesized: (a) Echavarren, A., Galán, A., de Mendoza, J., Salmerón, A. and Lehn, J.-M. Helv. Chim. Acta 71 (1988) 685; (b) Kurzmeier, H. and Schmidtchen, F. P. J. Org. Chem. 55 (1990) 3749.

7. Amidines and guanidines are known to be good catalysts for nitroalkanes in Michael reactions: (a) Ono, N., Miyake, H., Kamimura, A., Tsukui, N. and Kaji, A. Tetrahedron Lett. 23 (1982) 2957; (b) Ono, N., Kamimura, A. and Kaji, A. Synthesis (1984) 226; (c) Pollini, G. P., Barco, A. and De Giuli, G. Synthesis (1982) 44; (d) Andruszkiewicz, R. and Silverman, R. B. Synthesis (1989) 953.

8. Panunto, T. W., Urbánczyk-Lipkowska, Z., Johnson, R. B. and Etter, M. C. J. Am. Chem. Soc. 109 (1987) 7786.

9. (a) Schmidtchen, F. P., Gleich, A. and Schummer, A. Pure Appl. Chem. 61 (1989) 1535; (b) Müller, G., Riede, J. and Schmidtchen, F. P. Angew. Chem. 100 (1988) 1574; Angew. Chem., Int. Ed. Engl. 27 (1988) 1516; (c) Echavarren, A., Galán, A., Lehn, J.-M. and de Mendoza, J. J. Am. Chem. Soc. 111 (1989) 4994.

10. Schmidtchen, F. P. Tetrahedron Lett. 30 (1989) 4493.

11. Gleich, A., Schmidtchen, F. P., Mikulcik, P. and Müller, G. J. Chem. Soc., Chem. Commun. (1990) 55.

Received December 2, 1991. 